\title{
Selective Propagation of Social Data in Decentralized Online Social Network
}

\author{
Udeep Tandukar and Julita Vassileva \\ Department of Computer Science, University of Saskatchewan, Saskatoon, Canada \\ udeep.tandukar@usask.ca,jiv@cs.usask.ca
}

\begin{abstract}
In Online Social Networks (OSNs) users are overwhelmed with huge amount of social data, most of which are irrelevant to their interest. Due to the fact that most current OSNs are centralized, people are forced to share their data with the site, in order to be able to share it with their friends, and thus they lose control over it. Decentralized OSNs provide an alternative which allows users to maintain control over their data. This paper discusses an approach for propagation of social data in a decentralized OSN so as to reduce irrelevant data among users. The approach uses interaction between users to construct relationship model of interest. This relationship model acts as a filter later while propagating social data of the same interest group. This paper also presents a plan of a simulation to analyze our approach.
\end{abstract}

Keywords: Online Social Network, Decentralization, Peer-to-peer system, Information propagation, Relationship modeling

\section{Introduction}

Online Social Networks (OSNs) have become a common ground where people are generating and consuming huge amount of information. This information varies from personal thoughts (like status updates) to global news (such as wars, world cup, etc.). There have been growth in the number of providers of such OSNs and user's data are scattered over these different providers. In OSNs there is a huge flow of information of which only a fraction is relevant to the users. Since decentralized OSNs also inherit most of the issues from OSN, they have to deal with the question of how to provide relevant information to users and filter out the irrelevant information.

The currently popular OSNs are centralized which means they store all the information that people are generating or consuming. This information is mostly private and people voluntarily share it with the site, in order to be able to share it with their friends. However, in this way, they have less control over their own data and their data is scattered over the internet in different OSN providers which usually do not support data interoperability (apart from trivial user profile information).

This issue of control and privacy in centralized OSNs has recently motivated research into decentralized OSNs where the data of users are kept in their own clients (nodes). There have been several attempts to build decentralized OSNs and these 
projects are still going on [3], [7], [15]. Among several approaches to build decentralized OSN, a peer-to-peer ( $\mathrm{P} 2 \mathrm{P})$ infrastructure has also been proposed. $\mathrm{P} 2 \mathrm{P}$ has been popular among file-sharing applications, but not in OSNs. The inherent nature of how people connect with each other in a social network makes peer-to-peer architectures suitable for building decentralized OSNs.

This paper proposes an approach to deal with both problems mentioned above information overload and privacy - using a P2P infrastructure and relationship models according to interest groups among users to filter out irrelevant information from flowing out of the source. These relationship models are updated depending on feedback resulting from the interaction between users and what they do with the information they receive. The relationship models are used later to route the information that a user sends to her friends appropriately according to its semantic meaning.

The rest of the paper is structured as follows. A review of related works is presented in Section 2, followed by definition of our research problem in Section 3. Section 4 discusses our approach on filtering out irrelevant social data using relationship models. The proposed plan of simulation and evaluation of the discussed approach is presented in Section 5. Finally, Section 6 concludes the paper.

\section{Background and Motivation}

The section begins with an overview of online social networks and decentralized online social networks as an alternative to centralized ones. Then there is discussion about using peer-to-peer architecture for decentralized online social networks. Then we mention about information dissemination in social networks, and user modeling which is related to relationship modeling in our work. And the problem statement of our research is covered at the end of this section.

\subsection{Online Social Networks}

An Online Social Network (OSN) is defined as web platform in which a person can create a profile, connect to other people, view and traverse network of connections within the system, share resources and information within the system, and use social applications with which people within the system can interact and collaborate with each other [5], [9]. With the growth of internet usage, OSNs first came into existence in the form of SixDegree.com (in 1997) which had basic OSN features [5]. In the following years many more OSN service providers (like Friendster, MySpace, Last.FM, Hi5, Twitter, Facebook, etc.) came into existence, some of which have grown to have millions of active users. All these OSN followed client-server architecture in which the service provider is centralized. This architecture supports high accessibility since users can access the service from any web-browser wherever and whenever they desire. But due to this centralized nature, these OSN have inherent issues like single point of failure, central administration that can control activities of users, privacy issues due to central data storage, and requirement of larger servers and 
bandwidth to accommodate the growing number of users. With these issues in centralized OSN we saw discontinuation of some popular OSN services like SixDegree.com and Friendster [5], and the users lost all their social contacts and data when they lost access to those services. Some centralized OSN services like Twitter, due to growth in their popularity were having many performance scalability issues and still face some frequently slow response or even unresponsiveness [8]. In addition to these technical issues, there are also social issues arising with the rapidly growing popularity of social networking. People became more conscious about the information that they share in their social networks. Services, like Facebook which have millions of users, frequently have to deal with privacy issues. For example, Facebook's Beacon online ad system was tracking activities of the users in third party websites even when users were logged off from Facebook and had declined to broadcast their activities [14]. The system caused an outrage among Facebook users, and Facebook quickly discontinued Beacon. However, present centralized OSN still have full control over user data once shared, the user losses control over it and cannot remove it or export it into another OSN. Although there are various OSN available in web, there is no easy interoperability across them. Users of OSN (e.g. MySpace) cannot interact with users of another OSN (e.g. Facebook). This has lead OSNs being viewed as "information silos" [23]. As alternative to this centralized OSN, we can consider decentralized OSN in which users have control over their data.

\subsection{Decentralizing Online Social Networks}

Decentralized online social networks have distributed computing structure with trusted network of servers or peer-to-peer network. In [23], the authors suggest that decentralized OSN will give back to users the control of their data with respect to privacy, data ownership and information dissemination.

Users hosting their own social data. According to Yeung et.al [23], in decentralized OSN the user is not required to be a part of social networking services like Facebook, Twitter, etc. to maintain his/her online social presence. The user can host a FOAF [6] (Friend-Of-A-Friend) file, an activity log, photos/videos, and social client in a trusted server. They will have full control over whom and what to share out of his/her social data. The authors describe how the functionality offered by popular social applications, such as "Personal Wall", "Photos", and "News Feed" can be implemented in decentralized OSN. In the proposed system, the user shares and communicates social data with other users by using WebDAV [22] or SPARQL Update [18] protocols. As a prototype they have developed "Tabulator" [3] which is a generic data browser and editor of linked RDF (Resource Description Framework) data [4]. These types of decentralized OSN encourage users to store their social data on the web in standard format such as RDF and it should be accessible through URI (Universal Resource Indicator). Therefore, the user does not have to rely on only one social application, and can use any social application that support these open technologies. 
Using P2P infrastructure. Decentralized OSN can also be implemented with the use of Peer-to-Peer (P2P) network. A P2P network is a distributed network in which nodes are connected with each other to participate in processing, memory, and bandwidth intensive tasks. These networks scale better than centralized server architectures without the need of costly centralized resources. P2P networks have been popular mostly as file sharing networks (such as KaZaA, BitTorrent, etc.) and sometimes as collaborative sharing networks (such as Skype), but have not been used as a medium for online social networking. The inherent nature of peer-to-peer connection between users in a social network makes OSN suitable for peer-to-peer architecture [9]. In file sharing P2P systems like Gnutella, most of the users are free riders [1]. In contrast, $\mathrm{P} 2 \mathrm{P}$ applications like Skype, where users tend to stay connected to the network to receive calls from their friends, shows the potential that $\mathrm{P} 2 \mathrm{P}$ holds as implementation infrastructure for OSN.

To accommodate the familiar functionality of centralized OSN (like status updates, photo uploads, commenting, rating) in decentralized OSN, there are various challenges. Since the social data is stored at the peers, the availability of the social data depends on the online behaviour of peers. The storing of data on the peers allows encryption of these data which can ensure privacy while transmitting data from peer to another. The propagation of social data or updates among users in the OSN should be managed so that there is less duplication and no latency. These and other challenges have been discussed in detail in [9].

Some P2P systems have exploited properties of social networks (like trust, collaboration) in other to improve the performance of P2P networks. System like Tribler [15], has adopted social networking on BitTorrent based P2P file-sharing network in order to recommend, search, and download contents. The authors have used "Buddycast Algorithm" that exchanges preferences of peers in the implicitly defined social network to generate recommendation list and search contents. By using a collaborative download protocol called " 2 Fast", in which users collaborate to contribute their bandwidth, download performance also improved. Some also used the graph topology of real social networks in order to form a P2P overlay topology and used it to improve lookups and scalability [2].

PeerSoN [7] is an effort to build decentralized OSN over theP2P architecture. It has implemented encryption of user data to protect privacy and ensure direct exchange of data between devices for delay-tolerance and opportunistic networking. It uses OpenDHT [17], a Distributed Hash Table (DHT) service, for look-up service to find other peers in the P2P network and also to store data like, IP address, file information, and notifications for peers. In DHT [16], \{key, value $\}$ pairs are stored in a distributed nodes and node can retrieve the value associated with any key efficiently. The prototype of PeerSoN provides functionality like social links (becoming friends), storage to maintain profile and post by their friends, asynchronous messaging, and live chatting. 


\subsection{Information dissemination}

Nowadays, OSN produce huge amount of information and propagation of this information to its destination has to be well coordinated so as to reduce information overload, duplication, latency and to ensure quality.

In a file-sharing P2P network, the location of a resource is a very important piece of information. Generally, users send queries about resources of interest and system returns lists of their locations (i.e. peers that store these resources). In contrast to this process of searching, [13] discuss "selective information push" where user posts her profile to "super-peers" and receives notifications about resources that match her interests as these resources become available. Since the user is both consumer and producer of the resources shared in the network, she can also post advertisements of her resources and the super-peer will push notifications about these resources to relevant peers (peers with matching interests). This mechanism depends on the preferences of the user querying super-peers and if user has a more general interest then she might get lots of notifications. Here, the super-peer can be taken as a recommender that is pushing information according to the peer's interest.

The Push-poll recommender algorithm [20] propagates information through implicit social network, formed by peers with similar interests, using "word of mouth" mechanism. This system also takes in account feedback from the recipient to determine the future influence of sender on recipient. KeepUp recommender system [21] is based on the Push-poll algorithm. It allows user to interactively adjust the amount of influence that her neighbours have on the recommendations she receives. This gives power to user to decide indirectly what and how much information is propagated to her.

GoDisco [10] focuses on dissemination of social data according to the context of the information. The nodes gossip about their interests and strength of these interests with their neighbours in a regular interval. They also keep track of the behaviour of their neighbours (like activeness, forwarding behaviour). This knowledge of each other is used in the dissemination phase where the messages are assumed to have some semantic value that can be mapped to the interests of the nodes. Our work will consider the degree of relationship between these nodes and influence of this on dissemination. We will be creating relationship model of neighbouring nodes to determine degree of relationship.

\subsection{User Modeling}

Each user has her own characteristics, e.g. interests, preferences, etc., and we can utilize it to provide her with relevant social data in social network. These characteristics comprise the user model for the system. In our work, we will be building user models of the neighbours of a user within particular domain of interest in order to evaluate which information to forward to them or not.

Since the user will be using her OSN data on different devices and possibly across different applications, interoperability of the user models along with other social data is very important. That is why it is desirable for the representation of user data in the 
user model to follow some ontology so that it could be understood and interpreted outside of the context of the application in which the model was created. Using RDFbased user model like UserML [12] can enable distribution of the user model among different devices. UserML divides user model dimension in three parts: auxiliary, predicate and range. If we want to express the interest of a user in UserML then auxiliary will be "hasInterest", predicate will be "reading" to indicate her interest and range can be "low-medium-high".

To stimulate cooperation while sharing resources in P2P system, Sun et.al [19] has applied user modeling and modeling of relationships between users. With the help of user models of interest, they were able to route information to other users with similar interests. Using relationship modeling between users, they were able to determine typical time patterns of neighbour's behaviours to ensure better quality service. The authors created an overlay topology over the P2P network, where a relationship between users is created when a user successfully downloads a file from another user and the strength of the relationship grows with the number of successful interactions between these users.

With the increasing interest of users in social networking on web, there has been significant growth in research related to OSN. Users are becoming more and more sensitive to their data and decentralized OSN holds a key for these users to use web with full control over their data. As discussed earlier for decentralized OSN users can either choose secure server to host their data or use P2P infrastructure. As a new domain for P2P infrastructure, OSN holds lots of challenges to the researchers. As OSN is based on sharing of information, well-coordinated propagation of information is very important to handle information overload, latency, and repetitions. In our work, we will be focusing on using models of interest of neighbouring users for proper propagation of information.

\subsection{Problem Statement}

Online social network (OSN) has provided a medium for people to communicate and share information (social data). People share their thoughts, photos, videos, links to web pages, etc. in this network. The network in OSN constitute of members that are interconnected with each other through some relationship like friendship, common preferences, etc. When shared to the network, the shared social data propagate to each and every member of the network whether it is relevant to them or not. From the viewpoint of a sender, she is sharing only one social data at a moment. But from the viewpoint of a receiver, there can be more than one sender. According to statistics from Facebook [11], on average users have 130 friends. If all of the friends share a social data then a user will get 130 different social data. In this way, the user's stream is flooded with huge amount of social data, most of which are irrelevant to the user's interest.

Most of the available OSNs are based on client-server architecture in which user's social data are kept centralized. As discussed earlier this centralized nature has some issues and as an alternative we can have OSN in decentralized architecture, where users have control over their own social data. Even in decentralized OSN, due to the 
social nature of the network we will have to deal with propagation of social data to reduce irrelevancy, redundancy, and latency. The research domain in this paper is related to propagation of social data from a user to her neighbours so that they only get data relevant to them.

\section{Approach}

An approach of selective propagation of social data (i.e. information shared by users in social networks, such as status updates, shared links) by modeling interest of neighbouring users in a social network is proposed next, that ensures that social data reaches only the relevant users for whom it would be interesting.

\subsection{Social P2P Network of Users}

The system is a decentralized online social network implemented over P2P network. For simplicity, we will be dealing with a social network which can be represented by social graph. Social graph is a graph in which each user is a node and relationships between users is edges. Let $G$ be a social graph represent by $\{N, E\}$ where $N$ represents set of nodes (users) and $E$ represents set of edges (relationships) between nodes. We can say $n_{a} \in N$ have some relationship with $n_{b} \in N$ iff there exists $\left\{n_{a}, n_{b}\right\}$ or $\left\{n_{b}, n_{a}\right\} \in E$.

To route relevant social data to users, each user or node in the graph will model the interests of other users with whom she has relationships. From the point of view of a given user, the model of interests of other users is considered as relationship model since it signifies how many positive interactions have happened between the users in the context of particular area of interest. Positive interaction between two users in a given area of interest means that one user has sent social data related to the area of interest to the second user, and the second user has given positive feedback after receiving the social data. As a result of positive interaction, the strength of the relationship between the two users in the area of interest increases. The relationship model is used by the peer to adaptively disseminate social data related to a given interest area $I$, by sending it to peers with whom the user has sufficiently strong relationship in area $I$.

\subsection{Relationship modeling}

In an online world, relationships between users strengthen as the interaction between them increases. In the proposed approach, not only interaction between users in general but within certain subject or interest is taken into account, so that the system can model the strength of relationship in an area of specified interest between interacting users. To determine the area of interest of the social data, users have to either tag their updates with the interest areas or the system has to extract semantics from the data. 
Interaction between users within certain context is captured by tracking the feedback of the shared content. Feedback from friends (users connected in social graph) can be of different types. For the proposed system, feedback is categorized as follows.

Table 1: Categorization of feedback

\begin{tabular}{|c|c|c|}
\hline Type & Action & Value \\
\hline Type 1 & Comment / Share & 0.9 \\
\hline Type 2 & Rate / Like & 0.7 \\
\hline Type 3 & View / Open & 0.5 \\
\hline Type 4 & Ignored / Not open & 0.3 \\
\hline
\end{tabular}

The response value varies from 0.3 to 0.9 according to the type of action the users takes. These feedbacks from the receiver of the social data depend on the level of interest and relationship model with the sender for that context. The relationship model depends on the previous interaction between two users, and priority of a new social data is determined according to previous history of interaction in the system.

The relationship model consists of a list of areas of interest and the corresponding strength of relationship between two users in each of these areas. Strength of relationship between user $A$ and user $B$ for an interest area $I$ should increase with stronger feedback and decrease with weaker feedback, therefore it is calculated using following equation:

$$
S_{A}^{B}(I)=\alpha * S_{A}^{B}(I)_{P}+(1-\alpha) * F .
$$

Here, $S_{A}^{B}(I)$ is the new strength of relationship, $S_{A}^{B}(I)_{P}$ is the previous strength of relationship for an interest area $X$. The parameter $\alpha \in[0,1]$ is a linear function of the number of social data produced by the user in particular interest area $X$. Initially $\alpha$ is 0.9 so that the latter half of the equation has very low effect on the new strength. The feedback from the recipient is denoted by $F$, and its value varies from 0.3 to 0.9 as specified in Table 1. The increase and decrease of the strength of relationship calculated according to equation (1) is at very minimal rate, so as to maintain the relationship between the users as long as possible.

For the propagation of social data belonging to interest $I$, the strength of relationship between users should be more than a threshold value. Initially, this strength of relationships among all users is set as 1 and it will increase and decrease according to the interactions between users.

This approach of propagating social data takes into account the feedback of friends and uses this feedback to calculate strength of relationship, which is used in the future as a filter while sending the data of similar topic. It is taken in consideration that if information is relevant to a user, she will at least open that message and the feedback value is 0.5 which is more than critical value. If the information is irrelevant to a user, she will ignore it and hence the feedback value is 0.3 which is less than critical value and reduces the strength of relationship. This relationship models are all stored in the user's device since our system follows decentralized architecture. The process of filtration during propagation is done at the sender; therefore, some computation power 
is consumed at the sender side but network traffic is reduced and the friend's node does not have to do much of the filtration process.

As the strength of relationship for a particular interest $I$ in user $A$ for user $B$ fades away, user $B$ will not get any social data related to interest $I$ from user $A$. For user $B$ to get social data related to interest I from user $A$, she has to make relationship model in user $A$ between her and user $A$ stronger. User $B$ can send a social data to user $A$ related to interest $I$, this will show that user $B$ is becoming interested in $I$ and user $A$ will increase the strength of relationship as high as possible so that social data from her can reach user $B$. To give more control to users over their relationships, it is also possible to allow users to directly adjust the relationship strength with other users via an appropriate GUI, similar to the interactive influence adjustment deployed in the KeepUP Recommender System [21].

In this way, relationships between users will grow or fade away in context to certain interest groups. This will allow better communication between users since they do not have to deal with irrelevant social data.

\section{Evaluation Plan}

In order to evaluate the discussed approach of using relationship modeling to filter out irrelevant social data in a social network, a simulation of the system will be developed using synthetically generated social graph and real-world social graph from StudiVZ and Facebook. A random social graph with small world properties will be generated using JUNG ${ }^{1}$ (Java Universal Network/Graph) Framework as a synthetic dataset. Afterwards, two different real datasets will be used to generate the network and message streams - one from StudiVZ ${ }^{2}$ and one from Facebook ${ }^{3}$. Both have around 1 million users or nodes.

\subsection{Distribution of interest}

Possible areas of interest can be defined for users in hierarchical way by introducing general categories and sub-categories, so initially, to avoid widely separated interests in population the system will only have one level of general categories of interest, such as "sports", "news \& events", "politics", "personal status updates", "photos", "videos", "curiosities \& jokes". Interests are distributed exponentially over users in the social network with most common interests (in the currently most popular music, movies, etc.) taking large portion of population and less common interest (e.g. local sport) popular among small portion. The mechanisms to generate such skewed distributions are known: growth - people gain new interests with time, and preferential attachment - areas of interest that are already popular attract newcomers with a higher likelihood. The simulation will use these rules to

\footnotetext{
${ }^{1} \mathrm{http}: / /$ jung.sourceforge.net/index.html

${ }^{2} \mathrm{http}: / /$ studivz.irgendwo.org/

${ }^{3}$ http://odysseas.calit2.uci.edu/doku.php/public:online_social_networks\#available_datasets
} 
generate a realistic distribution of interests for a fixed set of interest semantic categories.

The system depends on the growth of relationship strength between users for particular interest. The feedback of each shared data is important to calculate this strength. This feedback depends on the interest level of the receiver of the social data. The system will simulate around 25 interest categories and these will be distributed to all of the nodes in the graph. The nodes will have different interest levels, $I \in[0,1]$, which signifies how much the user is interested on each category. When the users receives an update of an interest (semantic) category, the higher the interest level, the likelihood of feedback from Type 1 to 3 as illustrated in Table 1 increases by the users. The users who have lower interest levels in the category of the update will give Type 4 feedback. The distribution of the interest levels initially will be random. Since there will be some nodes which will have more connection that other nodes, there is probability of these nodes being interested in more interest groups. With the interaction on particular area, the value of interest level will also grow. These considerations will be taken into account while designing the proposed simulation.

\subsection{Propagation of social data}

The propagation of social data depends upon the relationship model of each simulated user. Initially the system will consist of equally distributed relationship model (equal value of relationship strength) so that propagation of social data at the initial stage of the system reaches all friends of the user. With the feedback from friends, these relationship models will either strengthen or weaken according to equation (1). The likelihood of type of feedback as illustrated in Table 1 depends on the interest level of the friends as discussed in earlier section. For simplicity, each social data which will propagate will also carry semantic meaning along with it. The semantic meaning will consist of types of interest group the social data belongs to. To simulate the phenomenon of users injecting new content in the system, each node in the system is fed with a number of social data within random interval of time. The behaviour of each node whether to forward (share) an incoming social data to its neighbour (friend) depends on the interest level, strength of the relationship with respect to the interest category of the social data represented in the relationship model. All these changes of relationship model, forwarding of data, filtering of irrelevant data and interest level will be recorded and used in future analysis.

\subsection{Analysis}

For each node, the following data will be recorded in the simulation:

- Relationship model established between each node with its friends.

- Interest level of each node.

- The number of social data a node shared with its friends.

- The number of social data filtered by relationship model in each node.

- The number of social data that is forwarded by a node. 
- The total number of nodes that forwarded social data received from its friends and interest level at the moment of forwarding.

With the simulation of our proposed system, we hope to have the following analysis and insights.

- New social data generated by a user will get filtered and the propagation will be limited by the relationship model. The rate at which the nodes evolve to filter out irrelevant social data will be analyzed.

- The level of interest in a user has direct impact on the type of feedback to a shared data. This feedback is used to calculate the relationship strength. The level of interest will change as the number of interaction increases or decreases. The correlation between interest level and relationship strength will be analyzed.

- A node can forward an incoming social data. This behaviour largely depends on the interest level. The system will record this forwarding behaviour of each node to analyze range of propagation of a social data. Range of propagation means how far a social data is forwarded from its source.

- Spreading or sharing of social data will largely depend on the interest level and relationship model of each node in the system. Sharing of a social data stops where node has low interest level or weak relationship model with her friends.

When the system reaches its maturity, it will have nodes interacting with each other without concerns about getting irrelevant social data.

\section{Conclusion}

In this paper, we have discussed an approach of using feedback from interaction between users as a relationship building mechanism to filter out irrelevant social data in decentralized online social networks. Decentralized online social networks give users the control of their social data with respect to privacy, data ownership and information dissemination. A simulation will be implemented to analyze the discussed approach. The simulation will also deal with the numbers of hops a social data transverse so as to analyze its spread. The simulation will have nodes which are always online and cooperative. But in the real system, there are always issues with availability, free riders, latency, and other factors. The area of decentralized online social networks holds exciting research questions, associated with storage of social data, privacy issues of social data, searching and indexing of friends in the network, and many more. We plan to implement a real decentralized OSN that follows P2P architecture after the successful analysis of the simulation and evaluation.

\section{References}

1. Adar, E. and Huberman, B.A.: Free Riding on Gnutella. First Monday 5 (10), 2-13 (2000)

2. Anwar, Z., Yurcik, W., Pandey, V., Shankar, A., Gupta, I., and Campbell, R.H.: Leveraging Social-Network Infrastructure to Improve Peer-to-Peer Overlay Performance: Results from Orkut. ACM Computing Research Repository (2005) 
3. Berners-Lee, T., Hollenbach, J., Lu, K., Presbrey, J., and Schraefel, M.: Tabulator redux: Browsing and writing linked data. In Proceedings of the 1st Workshop on Linked Data on the Web (2008)

4. Berners-Lee, T.: Linked Data, http://www.w3.org/DesignIssues/LinkedData.html (2006)

5. boyd, d and Ellison, N.B.: Social Network Sites: Definition, History, and Scholarship. Journal of Computer-Mediated Communication 13 (1), 210-230 (2008)

6. Brickley, D. and Miller, L.: FOAF Vocabulary Specification 0.98 , http://xmlns.com/foaf/spec/ (2010)

7. Buchegger, S., Schiöberg, D., Vu, L.H., and Datta, A.: PeerSoN: P2P social networking: early experiences and insights. In Proceedings of the Second ACM EuroSys Workshop on Social Network Systems, ACM, 46-52 (2009)

8. Cozzatti, J.P.: A Perfect Storm of Whales, http://engineering.twitter.com/2010/06/perfectstormof-whales.html (2010)

9. Datta, A., Buchegger, S., Vu, L.H., Strufe, T., and Rzadca, K.: Decentralized Online Social Networks. In B. Furht, ed., Handbook of Social Network Technologies and Applications, Springer, 349-378 (2010)

10. Datta, A. and Sharma, R.: GoDisco: Selective Gossip Based Dissemination of Information in Social Community Based Overlays. Distributed Computing and Networking, 227-238 (2011)

11. Facebook: Statistics, http://www.facebook.com/press/info.php?statistics (2010)

12. Heckmann, D. and Krueger, A.: A user modeling markup language (UserML) for ubiquitous computing. Lecture Notes in Artificial Intelligence 2702, 393-397 (2003)

13. Koubarakis, M., Tryfonopoulos, C., Idreos, S., and Drougas, Y.: Selective Information Dissemination in P2P Networks: Problems and Solutions. ACM SIGMOD Record 32 (3), 71-76 (2003)

14. Perez, J.C.: Facebook's Beacon More Intrusive Than Previously Thought, http://www.pcworld.com/article/140182/facebooks_beacon_more_intrusive_than_previou sly_thought.html (2007)

15. Pouwelse, J.A., Garbacki, P., Wang, J., et al.: TRIBLER: a social-based peer-to-peer system. Concurrency and Computation: Practice and Experience 20 (2), 127-138 (2008)

16. Ratnasamy, S., Francis, P., Handley, M., Karp, R., and Shenker, S. A scalable contentaddressable network. In Proceedings of conference on Applications, technologies, architectures, and protocols for computer communications, ACM, 161-172 (2001)

17. Rhea, S., Godfrey, B., Karp, B., et al.: OpenDHT: A public DHT service and its uses. ACM SIGCOMM Computer Communication Review 35 (4), 73-84 (2005)

18. Seaborne, A., Manjunath, G., Bizer, C., et al.: SPARQL Update, http://www.w3.org/Submission/SPARQL-Update/ (2008)

19. Sun, L., Upadrashta, Y., and Vassileva, J.: Ensuring quality of service in p2p file sharing through user and relationship modelling. In Proceedings of User Modelling UM03 Workshop on User and Group Models for Web-Based Adaptive Collaborative Environments, Johnstown, 57-66 (2003)

20. Webster, A. and Vassileva, J.: Push-poll recommender system: Supporting word of mouth. In Proceedings of User Modelling, UM2007, Corfu, Greece, Springer, 278-287 (2007)

21. Webster, A. and Vassileva, J.: The KeepUP recommender system. Proceedings of the 2007 ACM conference on Recommender systems, ACM, 173-176 (2007)

22. Whitehead Jr, E.J. and Goland, Y.Y.: WebDAV: A network protocol for remote collaborative authoring on the Web. In Proceedings of the sixth conference on European Conference on Computer Supported Cooperative Work, 291-310 (1999)

23. Yeung, C.A., Liccardi, I., Lu, K., Seneviratne, O., and Berners-Lee, T.: Decentralization: The future of online social networking. In W3C Workshop on the Future of Social Networking Position Papers (2009) 